\title{
Article \\ Observing Planetary and Pre-Planetary Nebulae with the James Webb Space Telescope
}

\author{
Raghvendra Sahai (1) \\ Jet Propulsion Laboratory, California Institute of Technology, 4800 Oak Grove Drive, Pasadena, CA 91109, USA; \\ sahai@jpl.nasa.gov; Tel.: +1-818-354-0452
}

Received: 29 April 2020; Accepted: 3 August 2020; Published: 12 August 2020

check for updates

\begin{abstract}
Most stars in the Universe that leave the main sequence in a Hubble time will end their lives evolving through the Planetary Nebula (PN) evolutionary phase. The heavy mass loss which occurs during the preceding Asymptotic Giant Branch (AGB) phase is important across astrophysics, dramatically changing the course of stellar evolution, contributing to the dust content of the interstellar medium, and influencing its chemical composition. The evolution from the AGB phase to the PN phases remains poorly understood, especially the dramatic transformation that occurs in the morphology of the mass-ejecta as AGB stars enter the post-AGB phase and their round circumstellar envelopes evolve into pre-PNe (PPNe) and then to PNe. The majority of PPNe and PNe deviate strongly from spherical symmetry. Strong binary interactions most likely play a fundamental role in influencing this evolutionary phase, but the details of these interactions remain shrouded in mystery. Thus, understanding the formation and evolution of these objects is of wide astrophysical importance. PNe have long been known to emit across a very large span of wavelengths, from the radio to X-rays. Extensive use of space-based observatories at X-ray (Chandra/ XMM-Newton), optical (HST) and mid- to far-infrared (Spitzer, Herschel) wavelengths in recent years has produced significant new advances in our knowledge of these objects. Given the expected advent of the James Webb Space Telescope (JWST) in the near future, we focus on future high-angular-resolution, high-sensitivity observations at near and mid-IR wavelengths with JWST that can help in addressing the major unsolved problems in the study of $\mathrm{PNe}$ and their progenitors.
\end{abstract}

Keywords: stars: AGB and post-AGB; stars: mass-loss; pre-planetary and planetary nebulae; dust; circumstellar matter; James Webb Space Telescope; techniques: infrared; techniques: high-angular resolution

\section{Introduction: The Extraordinary Deaths of Ordinary Stars}

The late evolution and deaths of a very significant percentage $(\sim 60-80 \%)$ of stars in the Universe are likely to be fundamentally affected by strong binary interactions ${ }^{1}$. Binary interactions in particular affect our understanding of stars in general, since such interactions affect important diagnostic observables of stars and stellar populations (e.g., mass-loss and evolutionary time-scales). A striking example of the effect of binary interactions on stellar evolution are planetary nebulae (PNe) (e.g., see [2]), which represent the bright end-stage of most stars in the Universe that evolve in a Hubble time (i.e., those with main-sequence masses of $1-8 M_{\odot}$ ). The progenitors of $\mathrm{PNe}-$ Asymptotic Giant Branch (AGB) stars-lose almost all of their stellar envelopes via extensive mass-loss (with rates as high as $\left.\sim 10^{-4} \mathrm{M}_{\odot} \mathrm{yr}^{-1}\right)$. This is followed by the formation of pre-PNe (PPNe) and $\mathrm{PNe}$

1 If we include interactions with giant planets ("hot Jupiters") (e.g., [1]), then this percentage becomes much higher. 
during the post-AGB stellar phase. Intriguingly, all PPNe and the majority (i.e., $\sim 80 \%$ ) of PNe, unlike the generally roughly round circumstellar envelopes around most AGB stars ${ }^{2}$, display a spectacular array of morphologies and geometrical complexities. These include elliptical, bipolar and multipolar shapes, nested geometrical structures (e.g., hourglass within hourglass structures), equatorial density enhancements (including rotating disks, torii), low-latitude jets, and central stars offset from the geometrical centers of the nebulae (e.g., [7] (ST98), [8,9]), etc. In addition to pre-PNe and $\mathrm{PNe}$, post-AGB objects include the so-called disk-prominent post-AGB or dpAGB objects ${ }^{3}$ [10], that harbor large, possibly rotating disks, and little or no extended nebulosities. There are many possible ways a companion can generate aspherical PPNe, PNe and dpAGB objects-for example, via common envelope evolution (CEE), grazing envelope evolution (GEE), or focusing of mass-loss toward the equatorial plane (Bondi-Hoyle or Roche-Lobe Overflow), and the associated formation of circum-companion or circumbinary disks and jets (ST98, [11-13]). Although single-star models to explain PNe, invoking fast rotation and/or magnetic fields ([14]) have been proposed in the past, these are not likely $([15,16])$.

The world's most powerful astronomical observatories such as HST, Chandra and XMM-Newton from space, and the IRAM 30 m, ALMA, SMA, PdBI and the VLA, from the ground (e.g., Astronomy and Astrophysics Decadal Survey Astro 2020: [17,18]), have been used extensively in order to characterize the physical properties of aspherical PPNe, PNe and/or dpAGB objects, in the quest to understand how such objects result from AGB stars. In this paper, we discuss how the next large telescope in space for astronomy, the James Webb Space Telescope (JWST), can be used to provide key observational diagnostics for understanding the mysterious deaths of low- and intermediate-mass stars.

\section{Using the James Webb Space Telescope (JWST) to Study PPNe and PNe}

JWST is a large infrared telescope with a $6.5-\mathrm{m}$ wide, segmented, primary mirror that will be launched on an Ariane 5 rocket from French Guiana on 30 March 2021. JWST is an international collaboration between NASA, the European Space Agency (ESA), and the Canadian Space Agency (CSA). The primary, consisting of 18 hexagonal segments (with a clear aperture of $25 \mathrm{~m}^{2}$ ), has to be unfolded and the segments co-phased in space. The telescope optics has to be kept shielded from the Sun, the Earth and the Moon-this will be achieved by a giant sunshield (approximately $22 \times 14 \mathrm{~m}^{2}$ ), and putting JWST into orbit at $\sim 1.5 \times 10^{6} \mathrm{~km}$ from the Earth at the L2 Lagrangian point. JWST's operational lifetime is limited by the propellant for station-keeping to be about 10 years (with margin); the JWST project will perform mission assurance testing of the flight system to guarantee five years of scientific operations starting at the end of the commissioning period six months after launch. Its wavelength coverage is $0.6-28.6 \mu \mathrm{m}$. JWST is diffraction limited at wavelengths $\gtrsim 2 \mu \mathrm{m}$, with a PSF FWHM of 0 ' 075 at $2 \mu \mathrm{m}$.

The sunshield allows the telescope to cool down to a temperature $<50 \mathrm{~K}$ by passively radiating its heat into space. Its three near-infrared instruments (NIRCam, NIRSpec, FGS/NIRISS: see below) will work at about $39 \mathrm{~K}$ through a passive cooling system. The mid-infrared instrument (MIRI: see below) will work at a temperature of $7 \mathrm{~K}$ using a cryocooler system.

\subsection{Instrument Summary}

JWST's suite of four instruments are listed below, together with their wavelength coverage (the organizations/institutions responsible for their construction) and a short summary of the science observing modes offered by each instrument. A visual representation of these capabilities is given in Figure 1:

2 here we are referring to the extended regions of these envelopes and not the innermost regions which have been found to be remarkably aspherical in some objects, and we are excluding some notable examples with extended aspherical structures such as V Hya, $\pi^{1}$ Gru, GX Mon, and OH $231.8+4.2$ (e.g., see [3-6]).

3 objects with a post-AGB central star, a compact central disk, and little or no extended nebulosity. 


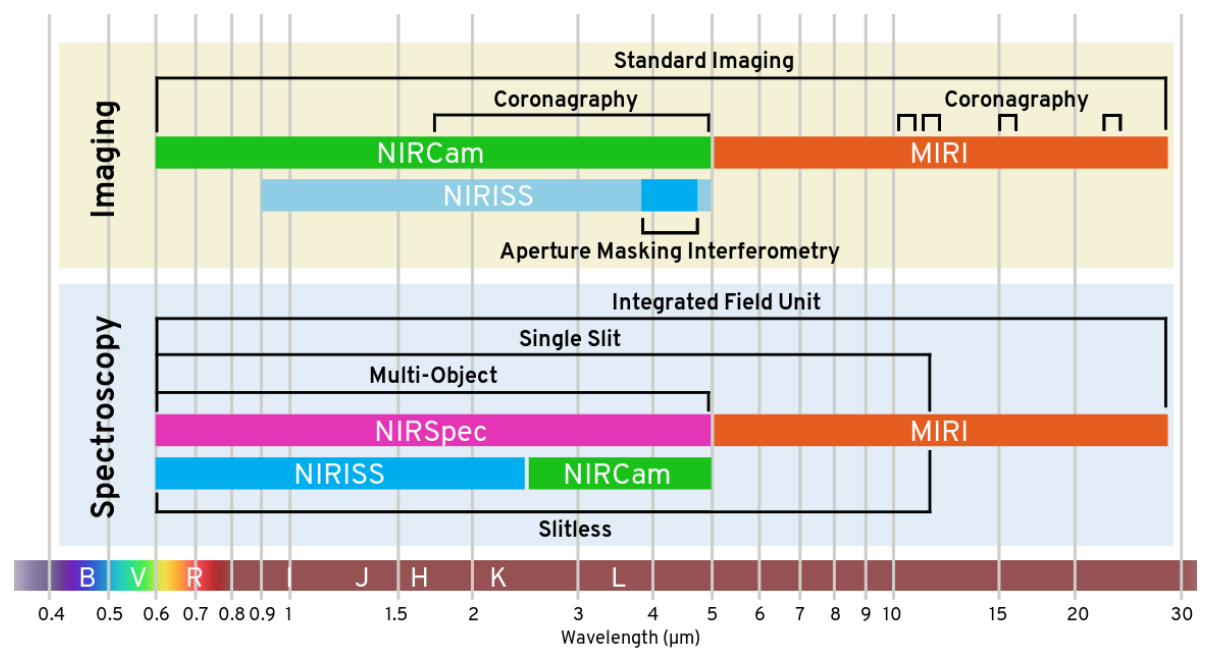

Figure 1. James Webb Space Telescope (JWST) instruments/capabilities at a glance (from http:/ / www.stsci. edu/jwst/instrumentation). Detailed information can be found at https://jwst-docs.stsci.edu/.

\subsubsection{NIRCam (Near Infrared Camera): $0.6-5 \mu \mathrm{m}$ (U. Arizona)}

NIRCam offers imaging, coronagraphy, and grism slitless spectroscopy. NIRCam has 2 modules pointing to adjacent fields of view. Each module uses a dichroic to observe simultaneously in a short wavelength channel $(0.6-2.3 \mu \mathrm{m})$ and a long wavelength channel $(2.4-5 \mu \mathrm{m})$. NIRCam has 5 observing modes for science: (i) imaging of two $2 .{ }^{\prime} 2 \times 2 .{ }^{\prime} 2$ fields separated by $44^{\prime \prime}$ covering $9.7 \mathrm{arcmin}^{2}$ in total (ii) coronagraphic imaging at multiple wavelengths (see Section 2.1.5), (iii) wide field slitless spectroscopy (2.4-5 $\mu \mathrm{m})$ using grisms with resolving power $R=\lambda / \Delta \lambda \sim 1600$ at $4 \mu \mathrm{m}$, (iv) time-series imaging (photometric monitoring), and (v) grism time series (spectroscopic monitoring), with $R=1600$ at $4 \mu \mathrm{m}$.

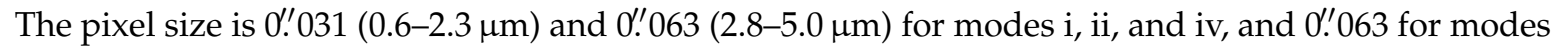
iii and v. NIRCam also serves as a wavefront sensor for aligning and phasing JWST's primary mirror.

\subsubsection{NIRSpec (Near Infrared Spectrograph): $<5 \mu \mathrm{m}$ (ESA, and NASA/GSFC)}

NIRSpec has 4 observing modes for near-infrared multi-object spectroscopy (MOS), integral field unit (IFU) spectroscopy, fixed slits (FS) spectroscopy, and bright object time-series (BOTS) spectroscopy, from $0.6-5.3 \mu \mathrm{m}$, as follows: (i) the MOS obtains simultaneous spectroscopy of multiple science sources within a $3.6^{\prime} \times 3.4^{\prime}$ field of view (FOV) using the micro-shutter assembly (MSA) ${ }^{4}$, (ii) IFU spectroscopy uses the integral field unit to provide spatially resolved spectroscopy over a $3^{\prime \prime} \times 3^{\prime \prime}$ FOV sampled with 900 pixels of size $0{ }^{\prime} 1 \times 00^{\prime} 1$, (iii) FS spectroscopy provides 4 fixed slits ( +1 for redundancy) for obtaining high sensitivity single object spectroscopy, and (iv) bright object time-series (BOTS) spectroscopy uses a 1". $6 \times 1$." 6 fixed slit aperture for observations of bright sources that require stable, high throughput time-resolved spectroscopy. The resolving power of NIRSpec varies from $\sim 100$ using a prism, to $\sim 1000$ (2000) $)^{5}$ using medium- (high-) resolution gratings.

2.1.3. FGS/NIRISS (Fine Guidance Sensor/Near-Infrared Imager and Slitless Spectrograph): $0.6-5 \mu \mathrm{m}(\mathrm{CSA})$

The FGS provides data for science attitude determination, fine pointing, and attitude stabilization using guide stars in the JWST focal plane. NIRISS provides 4 science observing modes that include slitless spectroscopy, high-contrast interferometric imaging, and standard imaging, covering a $2^{\prime} 2 \times 2^{\prime} 2$

4 individual shutter size is 0 ' $2 \times 0$ "' 46 in the dispersion direction $\times$ spatial direction; multiple shutters can be combined to form a slit.

5 these values apply at the central wavelength in the measured spectral range. 
FOV with a pixel size of $0^{\prime} \cdot 065$ These modes are: (i) wide field slitless spectroscopy (WFSS) with $R=150$ at $1.4 \mu \mathrm{m}$ over the entire field of view, using one or both of a pair of identical grisms and a selection of blocking filters to isolate specific wavelength intervals between $0.8-2.2 \mu \mathrm{m}^{6}$, (ii) single object slitless spectroscopy (SOSS) with a cross-dispersed grism with $R=700$ at $1.4 \mu \mathrm{m}$ that is designed to deliver broad wavelength coverage and spectro-photometric stability, optimized for time-series observations (TSOs), (iii) aperture masking interferometry (AMI) through specific filters that is enabled by a mask with seven sub-apertures (see Section 2.1.5), and (iv) standard imaging, that can be done in 7 wide- and 5 medium-band filters that are closely matched to the NIRCam filter set between $0.9-5.0 \mu \mathrm{m}$.

\subsubsection{MIRI (Mid Infrared Instrument): 5-30 $\mu \mathrm{m}$ (ESA and JPL)}

MIRI provides 4 observing modes for science: (i) imaging with 9 photometric bands $(\sim 5-12 \mu \mathrm{m})$ covering a $74^{\prime \prime} \times 113^{\prime \prime} \mathrm{FOV}$, (ii) coronagraphic imaging with four different filters (see Section 2.1.5), (iii) low-resolution spectroscopy $(\sim 5-12 \mu \mathrm{m})$ with a slit (size $0.51 \times 44^{\prime \prime} .7$ or slitless configuration, and (iv) medium-resolution spectroscopy $(\sim 4.9-28.8 \mu \mathrm{m})$ with four different IFUs, $\mathrm{R} \sim 1550-3250$. The pixel size for modes i to iii is 0 !'11, and 0 !'196 - 0!'273 for mode iv. MIRI can also be used effectively for parallel observations with other instruments.

\subsubsection{JWST High-Contrast Imaging}

JWST can perform coronagraphic observations with each of its 3 major science instruments:

(1) NIRCam, using (i) a round coronagraphic mask for observations at 2-4.4 $\mu \mathrm{m}$, with an Inner Working Angle [IWA] of $0^{\prime \prime} 4-0$ !' 81 , and (ii) a bar for observations at 2-3.7 $\mu \mathrm{m}$, with an IWA of $0 ! 13-0 . ' 88$.

(2) MIRI, using (i) a Lyot stop for observations at $23 \mu \mathrm{m}$, with an IWA of 2.16 and a $30^{\prime \prime} \times 30^{\prime \prime}$ FOV, and (ii) a 4-quadrant phase mask (4QPM) and filters at 10.65, 11.4, $15.5 \mu \mathrm{m}$, with an IWA of $0 !$ ' $33 \times 00^{\prime \prime} 49$.

(3) NIRISS, which admits light through seven holes or sub-apertures in an otherwise opaque pupil mask that interferes to produce an interferogram on the detector in the AMI, effectively making the full aperture of JWST into an interferometric array. The interferogram created by the aperture mask has a sharper core than is provided by normal "direct" imaging. The AMI mode allows the detection and characterization of a faint source within $\sim 0$ !' $07-0$ !' 4 of a bright one and up to $\sim 9$ magnitudes fainter. It can also be used to reconstruct high resolution maps of extended sources. The AMI mode uses one of 4 filters (three medium-band, one wideband) covering the $2.8-4.8 \mu \mathrm{m}$ range.

\subsection{JWST Strengths and Limitations for PPNe and PNe Studies}

JWST's major strengths for PNe and PPNe research are its ability to provide precise mid-IR imaging (due to its stable point-spread-function or PSF) with very high sensitivities. Although mid-IR observations can be done from the ground, the ambient thermal emission is a huge limiting factor, even at the short end of the mid-IR range: for example, for Keck, in the Lp band $(3 \mu \mathrm{m})$, the sky saturates the detector in a $>0.18 \mathrm{~s}$ exposure.

JWST's major limitations, for PPNe and PNe studies, are:

(1) Saturation (especially for Galactic targets), however mitigation strategies to deal with this to some extent are available and have been investigated by the Solar System science community (e.g., for enabling observations of Mars and the gas giants). Note though that none of the detectors can be damaged by bright light.

6 the grisms are mounted to disperse light in orthogonal directions on the detector. 
(2) Surveys of object classes that require slews from target-to-target will be expensive due to the long slew time $(\sim 30 \mathrm{~min})^{7}$.

(3) At the short-end of the NIR range, JWST faces competition from ground-based Large Telescopes with extreme Adaptive Optics (AO) capabilities. However, it is important to note that the Strehl ratio $^{8}$ for a ground-based telescope with $\mathrm{AO}$ depends on the seeing conditions, which can vary during the course of an observation, so observations of very faint extended circumstellar stricture around bright central sources can only be done with JWST.

\section{Selected Science Investigations}

Summarized below are several representative projects to study PNe and PPNe that exploit JWST's unique capabilities.

(a) Photometric and spectroscopic search for binary companions to, and/or disks around, the central stars of $\mathrm{PNe}$ (CSPNe), post-AGB, and post-RGB ${ }^{9}$ stars.

(b) Imaging faint and obscured circumstellar structures in PNe and post-AGB objects.

(c) Studying the composition and evolution of dust in galaxies.

(d) Studying the physics and chemistry of UV-irradiated molecular clumps in PNe.

\subsection{Binary Companions and Disks in Post-AGB and Post-RGB Objects}

If the formation and shaping of most $\mathrm{PNe}$ is a result of binary evolution (e.g., see review [12]), then the majority of CSPNe should have binary companions. Searches for such companions have been made using photometric (and radial velocity) variability over many decades starting with the early work by [19] that revealed companions in 10-15\% of a sample of $\sim 100$ CSPNe with periods, $\mathrm{P} \lesssim 3 \mathrm{~d}$. Recent searches have now considerably increased the sample size-with a dearth of objects at long periods ( $P>3 \mathrm{~d},[20-22])$. The small period (thus small orbital separation) of the binaries suggests that these objects must have undergone common-envelope (CE) evolution at an earlier phase of their evolution. Newer searches have used the infrared-excess as a signature of a cool (M-type, thus low-mass) companion-for example, the authors of [23] carried out I-band (and some J-band photometry) in 27 CSPNe and found 8 detections, but with low significance $(<2 \sigma)$; the authors of [24] observed $34 \mathrm{CSPNe}$, with a detection rate $32 \pm 16 \%$ (52 $\pm 24 \%$ ) in the I-band (J-band).

JWST can measure IR-excesses in the mid-IR with great precision, and can thus, compared to ground-based observations, find lower-mass companions in CSPNe and with a much greater success rate. For example, using MIRI imaging with different filters in the 5.6-28 $\mu \mathrm{m}$ range and a very modest integration time of $1000 \mathrm{~s}$ (that provides a $10 \sigma$ sensitivity of $0.63(2.2) \mu \mathrm{Jy}$ at $\left.5.6 \mu \mathrm{m}(10 \mu \mathrm{m})^{10}\right)$, and / or its low- and medium spectral resolution spectrographs, we can detect companions with masses as low as $0.2 M_{\odot}$, in CSPNe that are still very luminous (e.g., $L \sim 10^{3.4-3.78} L_{\odot}$, i.e., when they are at/near the knee of the WD cooling tracks [25]). Table 1 shows the companion to primary CSPN flux ratio, $R(m s / w d)$, at $\lambda=0.8,5.6$ and $10 \mu \mathrm{m}$ for 3 CSPNe at a distance of $3 \mathrm{kpc}$, spanning a representative range of effective temperatures (e.g., [26] find, for their list of 30 CSPNe in the LMC, that the majority have $T_{\text {eff }}$ values of $10^{4.8-5} \mathrm{~K}$, whereas about $20 \%$ have $T_{\text {eff }}>10^{5} \mathrm{~K}$ ). Including the source noise for the brightest WD at $10 \mu \mathrm{m}$ in the above table $\left(T_{\text {eff }}=10^{4.7} \mathrm{~K}\right)$, we find, using the JWST Exposure Time Calculator ${ }^{11}$, that we can detect the companion with $7 \sigma$ sensitivity. The strong increase in $R(m s / w d)$

7 This is the estimated slew time for a $53^{\circ}$ slew on-sky, adopted for use as a generic mean slew time in the Astronomers Proposal Tool. The actual slew time for a specific object may in general be shorter than this, because the telescope scheduling will attempt to minimize slews.

8 ratio of the peak aberrated image intensity from a point source compared to the maximum attainable intensity using an ideal optical system limited only by diffraction over the system's aperture.

9 like post-AGB objects, except that they have lost their stellar envelopes while on the RGB.

10 assuming that the source is sufficiently faint that its noise contribution is small compared to the instrumental noise.

11 https://jwst.etc.stsci.edu/. 
with wavelength clearly demonstrates the advantage of going to longer wavelengths for detecting the companion (the fluxes have been computed using black-body approximations for stellar spectral energy distributions in the mid-IR range.) An additional important advantage of working in the mid-IR range compared to the near-IR, is the significantly smaller effect of nebular/interstellar reddening.

Mid-IR excesses towards central stars may also arise from dusty disk structures, in addition to those due to the presence of a low-mass companion. Small disks ( $<0.01 \mathrm{AU})$, likely resulting from tidally disrupted asteroids, have been inferred from mid-IR excesses around many old, cool $(<24,000 \mathrm{~K})$ WDs (e.g., [27-29]). Larger disks ( 40-100 AU, i.e., Kuiper Belt size), perhaps resulting from collisions amongst dynamically rejuvenated Kuiper Belt Objects, have been found around central WD in the Helix Nebula, from Spitzer 24 and $70 \mu \mathrm{m}$ observations [30]. Spitzer observations of PNe ([31,32]) have revealed that 24/84 CSPNe visible in IRAC/MIPS images have IR-excesses (Figure 2). These excesses have been inferred to result from a (i) companion ( 2 objects), (ii) free-free and line emission from winds in [WC]-type central stars (7 objects), and (iii) dusty disk (13 objects). JWST, with a 7X smaller PSF and $\gtrsim 50 X$ higher sensitivity than Spitzer, will reduce nebular contamination and significantly increase the sample size that can be studied. By measuring the spectral-energy-distribution (SED) using MIRI imaging photometry and spectroscopy, together with NIRCam/NIRSpec observations in the near-IR, we will be able to search for the presence of both companions and disks. A strong correlation between the presence of companions and disks would imply that disk formation is related to the presence of the companion.

Table 1. Mid-infrared (IR) excess of a main-sequence (MS) companion ${ }^{1}$ in a central star of PNe (CSPN).

\begin{tabular}{|c|c|c|c|}
\hline WD & $M_{f}^{2}=0.54 M_{\odot}$ & $T_{e f f}=10^{5} \mathrm{~K}$ & $L=10^{3.4} L_{\odot}$ \\
\hline$\lambda(\mu \mathrm{m})$ & $R(\mathrm{~ms} / \mathrm{wd})$ & $F_{W D}(\mu \mathrm{Jy})$ & $\mathrm{F}_{M S}(\mu \mathrm{Jy})$ \\
\hline 0.8 & 0.0017 & 1938 & 3.2 \\
\hline 5.6 & 0.12 & 42.8 & 5.1 \\
\hline 10 & 0.155 & 13.4 & 2.1 \\
\hline WD & $M_{f}^{2}=0.58 M_{\odot}$ & $T_{e f f}=10^{4.7} \mathrm{~K}$ & $L=10^{3.78} L_{\odot}$ \\
\hline$\lambda(\mu \mathrm{m})$ & $R(\mathrm{~ms} / \mathrm{wd})$ & $F_{W D}(\mu J y)$ & $\mathrm{F}_{M S}(\mu \mathrm{Jy})$ \\
\hline 0.8 & $9.4 \times 10^{-5}$ & 33,880 & 3.2 \\
\hline 5.6 & 0.0063 & 810 & 5.1 \\
\hline 10 & 0.0082 & 257 & 2.1 \\
\hline WD & $M_{f}^{2}=0.58 M_{\odot}$ & $T_{e f f}=10^{5.2} \mathrm{~K}$ & $L=10^{3.45} L_{\odot}$ \\
\hline$\lambda(\mu \mathrm{m})$ & $R(\mathrm{~ms} / \mathrm{wd})$ & $\mathrm{F}_{W D}(\mu \mathrm{Jy})$ & $\mathrm{F}_{M S}(\mu \mathrm{Jy})$ \\
\hline 0.8 & 0.006 & 549 & 3.2 \\
\hline 5.6 & 0.44 & 11.7 & 5.1 \\
\hline 10 & 0.56 & 3.7 & 2.1 \\
\hline
\end{tabular}

An exciting new evolutionary channel for producing PPNe or dpAGB objects that requires binary interaction, but which occurs when the primary star is still on the Red Giant Branch (or RGB) has been highlighted in an ALMA study of the PPN, the Boomerang Nebula ([33] (Setal17)). The mass-loss that stripped the primary star of its envelope was driven at an extreme rate $\left(\sim 10^{-3} M_{\odot} \mathrm{yr}^{-1}\right)$ and expansion velocity $\left(165 \mathrm{~km} \mathrm{~s}^{-1}\right)$, much higher than found for any AGB star ([34]), and over a relatively short period (3500 yr), but the luminosity of the Boomerang's central star is much lower $\left(\mathrm{L} \sim 300 L_{\odot}\right)$ than possible for a post-AGB object. Setal17 found that the kinetic energy of the ejecta is extremely high, $>4.8 \times 10^{47} \mathrm{erg}$; thus mass-loss cannot be the result of the radiatively-driven dusty wind that characterizes the AGB phase, but is a result of a common-envelope (CE) ejection event which occurred when the primary was an RGB or early-AGB (E-AGB) star, thus explaining the low luminosity of the Boomerang's central star. A class of post-RGB objects similar to the Boomerang (i.e., with $L \lesssim 1000 L \odot$ ) 
have recently been identified in the Large and Small Magellanic Clouds (LMC and SMC), using optical spectroscopy and Spitzer ${ }^{12}$ photometry ([36-38]) photometry ([36-38]). Like the galactic post-AGB objects (i.e., PPNe and dpAGB), these post-RGB objects can be divided into shell-prominent sources (similar to PPNe) and disk-prominent sources (similar to dpAGB objects). Existing theoretical models of strong binary interactions for producing post-RGB objects such as CEE or GEE are still in their infancy (e.g., [39-41]), and one of the main reasons is the lack of observational data on the ejecta resulting from these interactions. Important diagnostics include the mass of material ejected in the equatorial plane (forming a disk), compared to that in a shell, and the chemical composition of the ejecta.
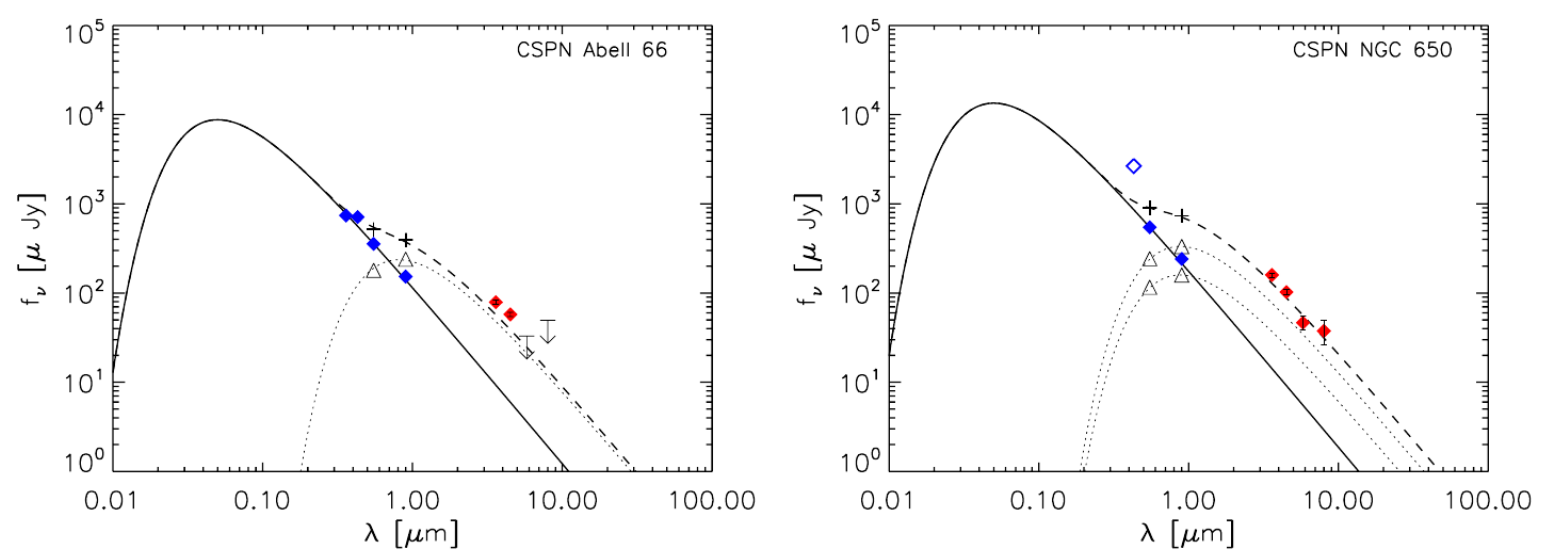

Figure 2. (adapted from [32]) Observed spectral-energy-distribution (SEDs) of two CSPNe with IR excesses caused by the companions' photospheric emission. Blue and red symbols are extinction-corrected fluxes (IRAC \& MIPS, in red, are extracted from the Spitzer images after removing the nebular background). The flux densities of the companion (triangles) and the sum of all components (plus symbols) are also shown. The solid (dotted) blackbody curve approximates the primary's (companion's) photospheric emission, and the dashed curves represent the sum of all emission. For NGC 650, the (visual) companion itself is a binary (the B magnitude is possibly inaccurate, hence shown as open diamond) and may not be associated with the CSPN.

Using MIRI/MRS on JWST, we can obtain 5-28 $\mu$ m spectra of a select sample of post-RGB stars in the LMC and SMC, and thus determine the chemical composition of the dust in the post-RGB objects. Post-RGB objects are expected to show O-rich chemistry, because He-shell burning and the third dredge-up (responsible for making C-rich stars) have supposedly not occured in RGB stars. However, recently, [42], from an analysis of FORCAST/GRISM spectra of a sample of RV Tauri stars (these belong to the class of dpAGB objects), find both carbon-rich and oxygen-rich dust in all objects in their sample, and conclude that the most likely scenario for this is CEE-the dual-chemistry mineralogy has been produced in a CE environment. The MIRI spectra will also constrain the abundance of crystalline silicates - these are generally lacking in the sample studies by [42], except for AC Her, where these have a relatively high abundance [43]. These differences may be related to the specific evolutionary channel (e.g., AC Her is believed to be a product of Roche-lobe overflow). It will be interesting to compare the chemical composition of dust in the post-RGB with post-AGB objects, since the bulk of the material in the extended ("shell") component in a post-AGB object is the result of a slow wind that operates over $\sim 10^{5} \mathrm{yr}$, and the dust formation conditions are very different than for post-RGB objects, where the CEE produces an outflow over very short timescales (few $\sim \times 10^{3} \mathrm{yr}$ ).

Using a simple 1D modeling approach, with a maximum of two components (as in [44]) -an extended shell and a compact inner component ${ }^{13}$ — SS20 have fitted the broad-band photometry of a

\footnotetext{
SpitzerSpaceTelescope [35].

13 the modeling strategy adopted by SS20 is conservative, beginning with a 1-component model and then if significant discrepancies remain, proceeding to a 2-component model. Note that the optical-to-mid IR SED is dominated by emission
} 
small sample of post-RGB stars and find that rough constraints can be set on the physical properties of the emitting component(s) (e.g., temperature distribution, dust masses, and the sizes of components that contain the emitting masses) (Figure 3). We have also made a preliminary 2D model with astronomical silicate grains of sizes $0.01-0.3 \mu \mathrm{m}$ to fit the broad-band photometry of the Boomerang, and found that at $10-25 \mu \mathrm{m}$, more than $99 \%$ of the total flux comes from an inner region $2 . / 5$ in diameter, consistent with the imaging data, which shows a warm, bright compact central disk structure (Setal17). These studies demonstrate that the MRS 5-28 $\mu \mathrm{m}$ spectrum (together with broad-band photometry, as necessary) will provide specific information about the dust composition, and enable one to constrain 2D models.

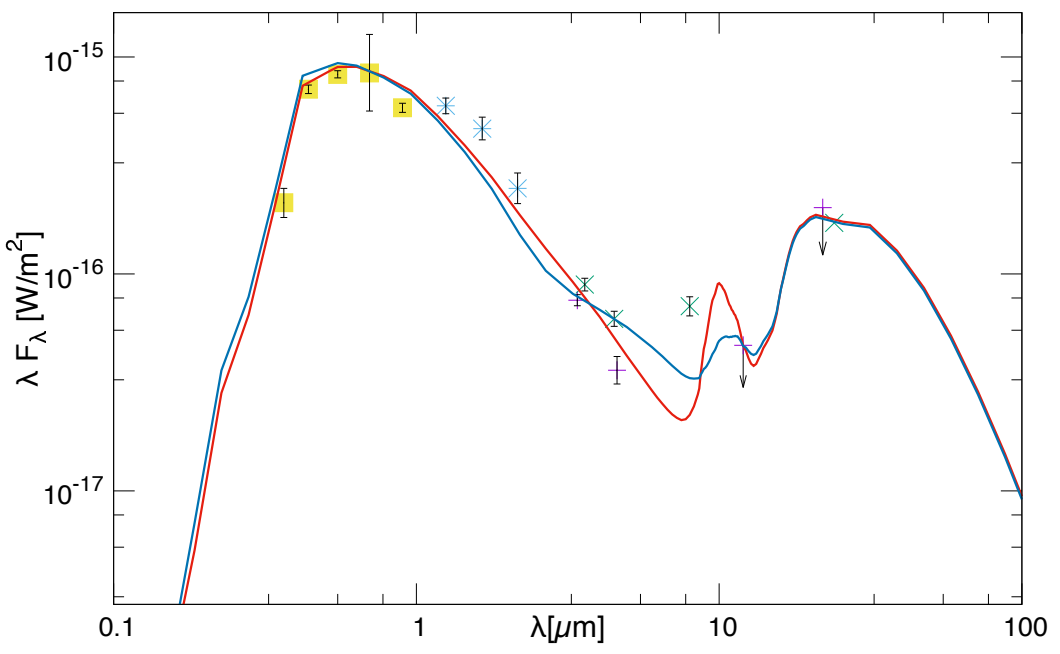

Figure 3. Observed spectral-energy-distribution of the post-RGB object, J043919.30-685733.4 and preliminary models. The observed fluxes are de-reddened for Galactic and LMC reddening. Curves show two preliminary "best-fit" two-component model fits using "warm" (Sil-Ow) silicate dust (optical constants from [45]). Each model includes an extended shell and a compact disk component, the former (latter) mainly constrained by the SED in the $\gtrsim 10 \mu \mathrm{m}(\lesssim 10 \mu \mathrm{m})$ range. The shell/disk mass ratio in Model 1 is higher than that in Model 2 by a large factor $(\gtrsim 10)$. In Model 2 the disk has large grains ( $a_{\min } \sim 1 \mu \mathrm{m}, a_{\max } \sim 25 \mu \mathrm{m}$ ), whereas the shell has standard MRN (i.e., small) grains. In Model 1, both the shell and the disk have standard MRN grains. The main discrepancies in the fits provided by these models are in the $\sim 5-12 \mu \mathrm{m}$ region, which may be due to the presence or lack of dust-spectral features (e.g., presence of PAH (Polycyclic Aromatic Hydrocarbons) emission in model 1, lack of silicate emission in Model 2) (adapted from Sarkar \& Sahai 2020, in preparation: SS20)-MIRI spectroscopy in the $5-28 \mu \mathrm{m}$ region is needed to determine if dust features affect the broad-band photometry and better constrain the modeling.

\subsection{Imaging Faint and Obscured Circumstellar Structures in Post-AGB Objects}

JWST's coronagraphic capabilities with NIRCam and MIRI, due to their relatively large IWAs, limit how close one can probe circumstellar matter around a bright central star of a PPN or a PN. However, Aperture Masking Interferometry (AMI) using NIRISS ([46]), in which most of the primary aperture is masked, leaving a set of holes that produce a set of 21 non-redundant baselines, can be used to detect faint structures of simple geometry close to bright sources - the resulting IWA is 0.'054 - 0.'094. Since this mode is designed to detect the thermal emission of young massive planets around bright stars, it is potentially sensitive to detecting expected faint structures (e.g., equatorial rings, disks) as

from a warm central component, whereas the far-IR SED is dominated by emission from an extended cool component, and since the latter is generally optically thin, its shape is unimportant. 
well as unexpected ones, around the central stars of PPNe and PNe-simulations by [47] demonstrate NIRISS's capability for various sky geometries (Figure 4).

Bipolar and multipolar PPNe show extended lobe structures and harbor dense, dusty, equatorial disks or torii. Their properties (physical structure, mass, kinematics) are intimately linked to the physical process(es) that terminate AGB evolution. The dusty disks/torii are generally optically thick at optical and near-IR wavelengths, and at least for a few objects with very high mass-loss rates, even the lobes can be optically thick at $\lambda \lesssim 2 \mu \mathrm{m}$-observations at $\sim 10 \mu \mathrm{m}$ are necessary to view the critical structures hidden within the lobes.

For example, in the bipolar "water-fountain" PPN, IRAS 16342-3814, the lobes, which showed smoothly varying brightness distributions in HST optical images, show a limb-brightened appearance in Keck/AO images at $\lambda \sim 1.6-3.8 \mu \mathrm{m}$ (Figure 5), and a remarkable corkscrew structure inscribed on the lobe walls is seen in the $3.8 \mu \mathrm{m}$ image [48]. The corkscrew structure has been interpreted to arise from the presence of a precessing jet, actively sculpting the bipolar lobe [48]. JWST's stable PSF offers a major advantage over current ground-based AO systems in imaging such structures. High signal-to-noise ratio images obtained at $>7.7 \mu \mathrm{m}$ using a dither-pattern, where JWST has an oversampled PSF, will allow for robust deconvolution and thus an improvement in the angular resolution beyond the diffraction limit ("super-resolution") for high S/N images. Ground based mid-infrared surveys of pAGB objects have already revealed the potential for such deconvolution (e.g., [49]). ALMA (interferometric) imaging reveals two collimated high-speed molecular outflows in IRAS 16342-3814, as well as an equatorial expanding ring [50]. Similar ring structures have been found in other objects as well, for example, in the well-studied Butterfly Nebula, M 2-9, [51] find two equatorial rings of cold molecular gas formed during short mass-loss episodes ( $\sim 40 \mathrm{yr}$ ), separated by $\sim 500 \mathrm{yr}$, inferred to result from the presence of a close, low-mass $\left(\sim 0.1-0.2 M_{\odot}\right)$ binary companion in a $\sim 90$ yr period orbit.
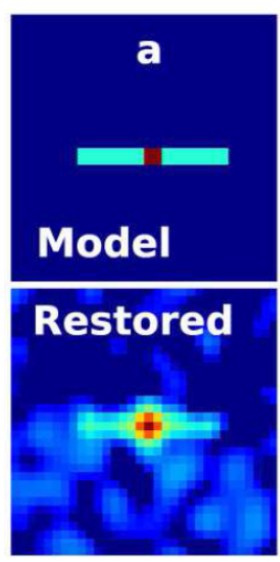
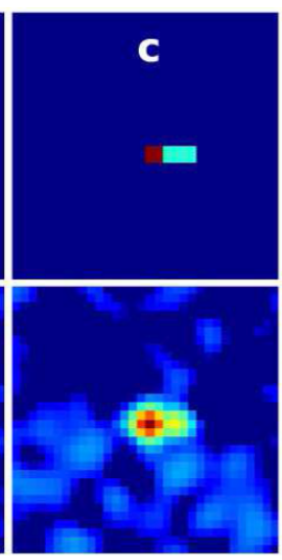
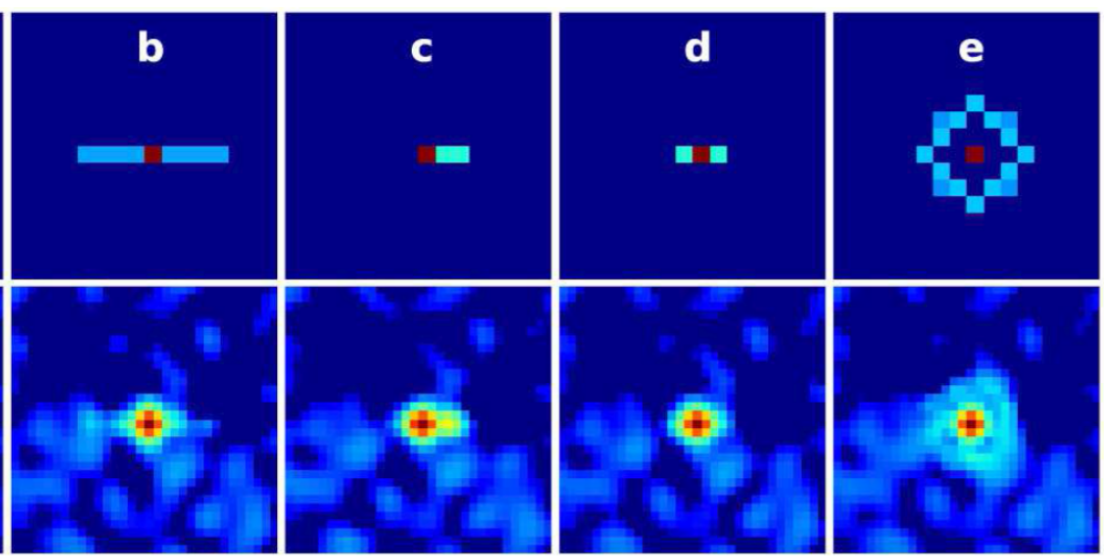

Figure 4. Input models and restored maps using NIRISS aperture masking interferometry (AMI) observations that combine two observations oriented perpendicularly (such orientations would be spaced apart by $\sim 3$ months) for a variety of sky geometries (adapted from [46]). The simulation suggests that a 100:1 pixel-to-pixel contrast is easily reachable with image reconstruction. (a) The integrated flux from the bar is 1 mag fainter than the point source. (b) The bar is 2 mags fainter than the point source. (c) An asymmetric 3-pixel bar with the same surface brightness as the bar in (a), and (d) a similar symmetric bar. (e) A 5-pixel diameter circular ring with integrated flux 1 mag fainter than the point source.

JWST imaging can reveal the dusty counterparts of such structures with high-fidelity for a select sample of PPNe. It may also find new structures not seen at other wavelengths. For example, Reference [52] find a pair of beautiful dusty rings surrounding the irregular PN, NGC 1514 in a $22 \mu \mathrm{m}$ (WISE) image of this object (Figure 6). Such rings or other extended cool, dusty, structures 
probably exist around other PNe as well. It is notable that no theory exists as yet to explain these ring structures!
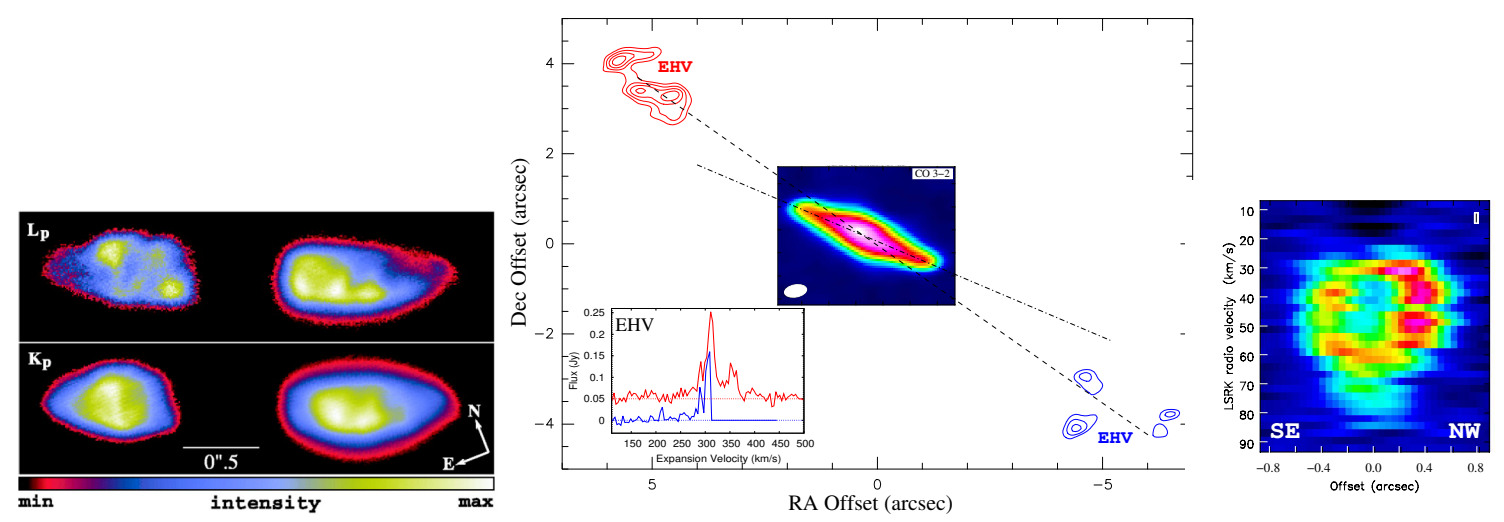

Figure 5. The water-fountain pre-PNe (PPN), IRAS 16342-3814. (left) (adapted from [48]) False-color Adaptive Optics (AO) images of IRAS 16342-3814 in the Lp and Kp bands. The right half of the image, covering the west lobe (which is significantly brighter than the east lobe), has been scaled differently in order to show structures in both lobes clearly. (middle) (adapted from [50]). Two high-velocity outflows seen in ${ }^{12} \mathrm{CO}$ emission - the "HVO" with an expansion speed of $\sim 260 \mathrm{~km} \mathrm{~s}^{-1}$ (colorscale), and the "EVO" (contours), with an expansion speed of $\sim 310 \mathrm{~km} \mathrm{~s}^{-1}$ and a different ejection axis than the HVO. Inset shows the spectrum of the red- and blue-shifted components of the EHVO, and the dashed (dash-dotted) line shows the axis of the EHVO (HVO). (right) (adapted from [50]) position-velocity cut along the major axis of the central ring structure.
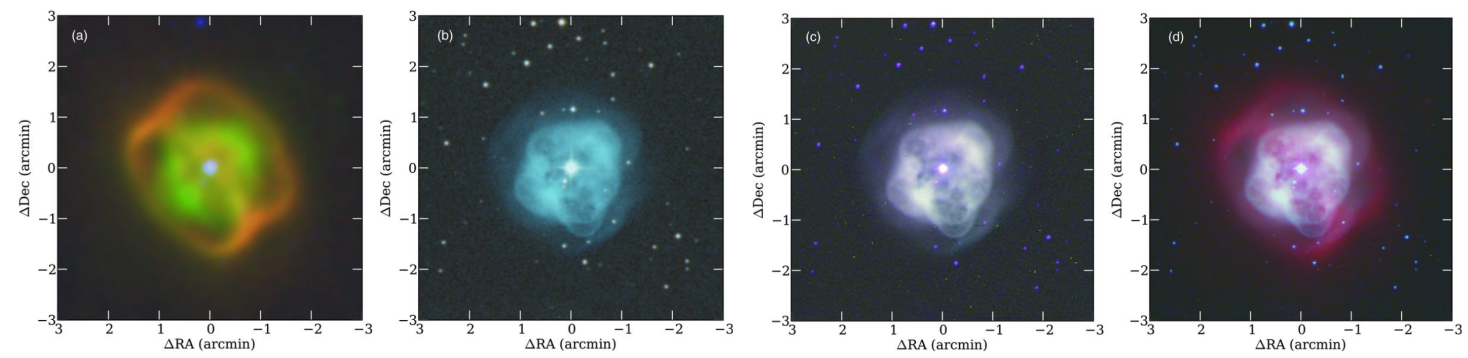

Figure 6. (adapted from [52]) The PN, NGC 1514 as seen with WISE and visible wavelength filters.

(a) WISE 3-color image, blue $=\mathrm{W} 2(4.6 \mu \mathrm{m})$, green $=\mathrm{W} 3(12 \mu \mathrm{m})$ and red $=\mathrm{W} 4$ (deconvolved) $(22 \mu \mathrm{m})$.

(b) Visible 3-color image derived from POSS B, R, and I images (blue $=B$, green $=B+R$, red $=R+I$.

(c) Visible wavelength combination of blue $=$ POSS B, green $=[\mathrm{OIII}]$, and red $=\mathrm{H} \alpha$. (d) Combination of blue $=$ POSS $\mathrm{B}$, green $=\mathrm{H} \alpha$, and red $=\mathrm{W} 3$.

Imaging of a sample of PPNe and PNe with MIRI would thus allow us to better probe the mass-ejection processes that terminate AGB evolution and lead to the dramatic change in morphology that these objects represent. A caveat to such observations is saturation, thus targets have to be chosen carefully, with the aid of dust radiative-transfer modeling that can provide surface brightness estimates. Of course, some objects may contain very small, clumpy regions of high surface brightness (i.e., microstructure) that cannot be predicted a priori (e.g., see HST image of the PN IC 418 (PK 258-00\#1) ([9]), and it is possible that these will be partially saturated in the MIRI images.

\subsection{The Composition and Evolution of Dust in Galaxies}

It is becoming increasingly important to understand the effect of metallicity on the physics and chemistry of the constituent stars and the ISM of galaxies. Different metallicities can result in different compositions for dust (and molecules), changing the spectra of stars and the ISM as well as those of the integrated light of galaxies. Low-metallicity environments are of special interest, as they provide a proxy for the ISM of galaxies in earlier epochs of the Universe. The 
formation, composition and evolution of dust features has been studied in galactic and extragalactic environments (LMC, SMC) (e.g., [53-57]). Amorphous carbon is thought to be the major constituent of circumstellar dust in carbon-rich stars on the $\mathrm{AGB}^{14}$ - these stars are the major contributor of such dust to the ISM. When such stars evolve to the PN phase, we find evidence for the presence of complex organic dust (both aromatic and aliphatic), which is likely produced during the pre-PN phases ([58]) (Figure 7). A well-known series of infrared (IR) emission features at 3.29, 6.2, 7.7-7.9, 8.6, 11.3, and $12.7 \mu \mathrm{m}$ dominate the dust emission, and are believed to be produced by PAHs (Figure 7). Many of the other mid-IR features have been identified with different kinds of dust such as fullerenes, $\mathrm{SiC}$, and MgS (Figure 7). Oxygen-rich objects show the presence of amorphous and crystalline silicates and ices. Some prominent narrow forbidden lines from ionized gas are also seen in the mid-IR spectra of PNe. With MIRI, one can carry out (a) more extensive spectroscopic surveys in the LMC and SMC than was possible with Spitzer, and (b) new spectroscopic surveys in nearby, metal-poor galaxies such as the Fornax dwarf spheroidal at 140 pc, of pAGB objects (PPNe, dpAGB and PNe).
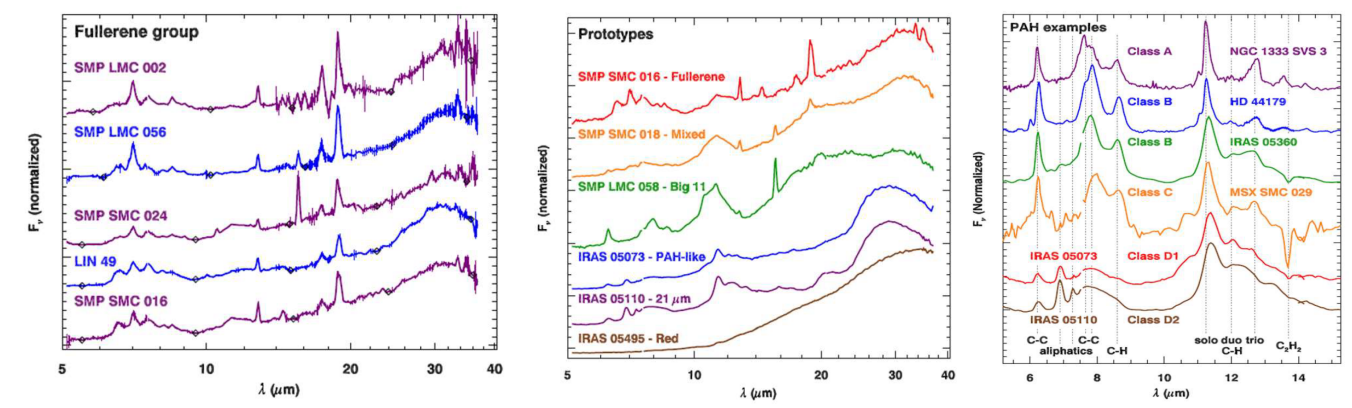

Figure 7. Carbon-rich dust features in pAGB objects in the Magellanic Clouds (adapted from [56]). (left) Spectra of fullerenes; (center) examples of the spectral groups into which carbon-rich pAGB objects have been grouped; (right) examples of spectra showing different classes of Polycyclic Aromatic Hydrocarbons (PAH) emission. Vertical dotted lines mark aliphatic modes at 6.9 and $7.27 \mu \mathrm{m}$, aromatic modes at other wavelengths, and $\mathrm{C}_{2} \mathrm{H}_{2} \mathrm{Q}$-branch absorption at $13.7 \mu \mathrm{m}$.

PAHS and fullerenes (C60, C70), which were first detected in the mid-IR spectra of PNe ([53,59]), are two very important and widespread constituents of the ISM. PAHs play a key role in the heating, chemistry and ionization of the interstellar gas [60]. The recent landmark association of fullerenes with several diffuse interstellar bands (DIBs, e.g., [61,62]) has provided the first definitive identification of a DIB carrier (a long-standing problem in astrophysics). In a recent study, the authors of [63] suggest that fullerenes may form in a top-down scenario, i.e., along a pathway in which larger molecules (with more than $60 \mathrm{C}$-atoms, e.g., large PAHs such as $\mathrm{C}_{66} \mathrm{H}_{48}^{+}$) transfer into smaller derivatives (including fullerenes) due to UV irradiation and a substantial number of the 500+ unassigned DIBs could be due to these derivatives.

In order to understand the formation of PAHs and fullerenes, it is necessary to map the spectral features from these species at high spatial resolution in $\mathrm{PNe}$, since for these objects, the radiation field and density structure are well-defined. For fullerenes, Spitzer observations have provided a sample of galactic PNe where these features been found [64], such as Tc 1 [53]. In this object, Gemini observations at $10 \mu \mathrm{m}$ already show that the ionized gas, the continuum-emitting dust, and the fullerenes have spatially distinct locations (Figure 8). MIRI/NIRSpec IFU mosaics can trace the emission from the central star to the fullerene-emitting region (a ring in Tc 1) and complementary MIRI images can sample the entire PN. The JWST IFUs unique combination of angular resolution, sensitivity and spectral coverage will allow one to pinpoint the spatial distribution of fullerenes in relation to other species (e.g., PAHs, species responsible for mid-IR plateaus), and derive the local

14 minor constituents could include graphite, hydrorgenated amorphous carbon, or soot-like particles. 
physical conditions (gas temperature and density), enabling tests of proposed fullerene formation mechanisms. For example, the MIRI and NIRSpec IFUs offer the prospect of resolving small-scale structures (such as disks) with potentially different dust emission features and trace the variations in these as a function of location in the nebulae-such data would then provide strong constraints on the formation and evolution of carriers of the different spectral components.

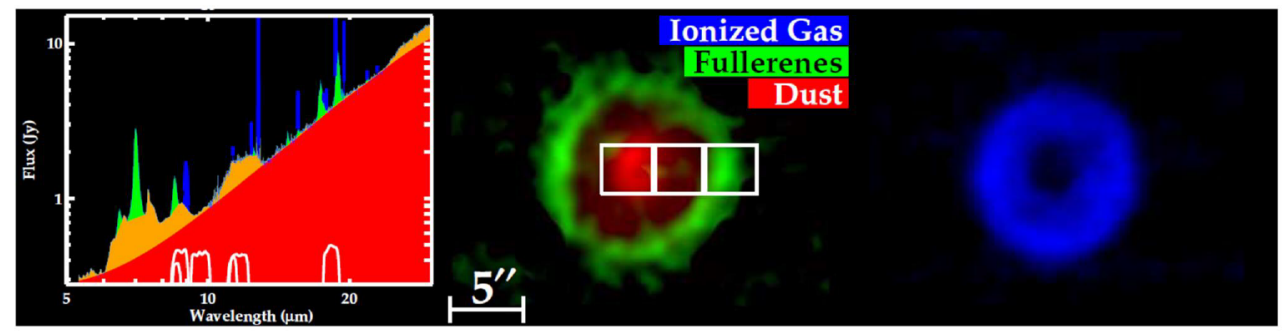

Figure 8. (left) Spitzer spectrum of Tc1; 6 images using Gemini/T-ReCS filter bandpasses (white curves) were processed with a filtered imaging decomposition tool, to create the false-color images showing the spatial distribution of fullerenes and dust (center), and ionized gas (right), color-coded to match the colors in the spectrum. Orange spectral features include mid-IR plateaus and PAHs. White rectangles show the size of the MRS mosaic footprint (J. Cami, priv. comm.)

\subsection{Study of the Physics and Chemistry of UV-Irradiated Molecular Clumps in PNe}

PNe provide a well-defined environment for studying the astrophysical problem of the survival of molecules and small dust grains in clumpy UV-irradiated environments (such as the photodissociation regions of massive star forming regions and AGNs, [65]. In evolved PNe, molecules are found in dense knots located inside the ionized region. The knots in PNe have been revealed via high-angular resolution HST optical, and $2.12 \mu \mathrm{m} \mathrm{H}_{2}$ imaging. Spitzer observations reveal that the PAH emission at $11.2 \mu \mathrm{m}$ in the well-studied PN, NGC 6720 (the Ring Nebula) is globally associated with $\mathrm{H}_{2}$ [66], but lack the spatial resolution to confirm whether the PAHs are directly associated with the knots, a necessary first step in testing PAH formation mechanisms. Observations of a few key PPNe with knotty structure such as NGC 6720, NGC 2440 and IC 4406 using MIRI IFU and NIRSpec IFU 15 observations at selected positions in the nebulae-including those that focus on individual globules (few arc seconds in diameter) and others that probe the main PDR in these objects (Figure 9)-together with narrow-band imaging of the main nebular region using NIRCam and MIRI, will, for the first time, spatially resolve the globules in the mid-IR, and enable mapping of the ionized, neutral and molecular regions within and around the $\mathrm{H}_{2}$ globules. JWST will detect multiple emission features from both the irradiated and cooler sides of the globules, including $\mathrm{H}_{2}$ ro-vibrational lines and PAH emission (in contrast, molecular line observations with ALMA can only detect the coldest, most shielded components of the globules). Emission lines from species such as [S III], [Fe III], and [Ne III,V] will be used to diagnose the physical parameters of (and abundances in) the ionized emitting gas surrounding the globules. The unprecedented sensitivity of JWST can potentially also result in detections of fine structure emission lines from trans-iron neutron-capture elements such as [Kr III,V], [Se III,IV], and [Cd IV] in the near-IR, unique probes of s-process nucleosynthesis during AGB evolution, which is responsible for the production of around half of the heavy elements beyond Fe in the cosmos [67].

15 the NIRSpec MSA can also be used. 


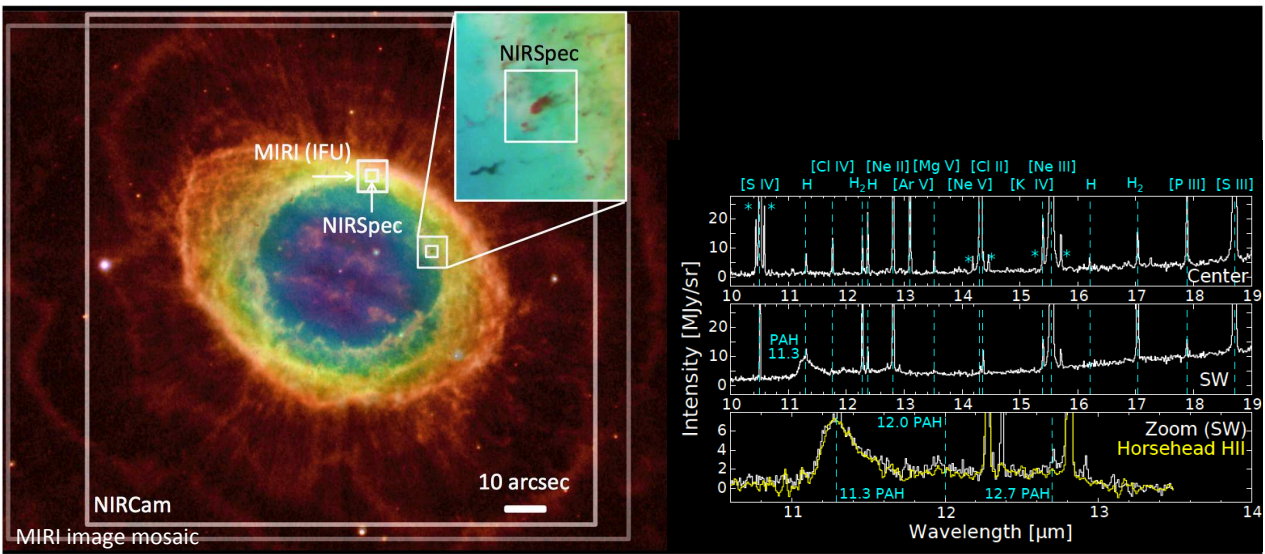

Figure 9. The PN, NGC 6720, (left) showing its knotty structure in a composite image combining optical imaging with HST in narrow emission-line filters F469N (He II, purple), F502N (O III, blue), and F658N (N II, green), and an LBT $2.12 \mu \mathrm{m} \mathrm{H}_{2}$ (red) image, together with various JWST instrument mode fields of view are superimposed (NIRSpec boxes represent IFUs); (right) Spitzer mid-IR spectra of two regions-near the center (top spectrum) and the main dust ring (middle spectrum), compared to that of the Horsehead Nebula (bottom spectrum), a well-studied PDR in the Orion Molecular Cloud complex with prominent PAH features (M. Matsuura, private communication).

\section{Summary}

Most stars in the Universe that leave the main sequence in a Hubble time will end their lives evolving through the Planetary Nebula (PN) evolutionary phase, and the accompanying heavy mass loss during their death throes is important across astrophysics-however the evolution from the red giant to the PN phase remains poorly understood. High-angular-resolution, high-sensitivity spectroscopic and imaging observations with JWST, especially at mid-IR wavelengths, will help in addressing the major unsolved problems in the study of PNe and their progenitors (post-AGB and post-RGB objects). Representative investigations that exploit JWST's unique capabilities include searches for low-mass binary companions to, and/or disks around, the central stars of PNe, post-RGB and post-AGB stars, the imaging of faint and/or obscured circumstellar structures in post-AGB objects, and studies of the composition and evolution of dust in galaxies.

Funding: This research was funded by in part by NASA through grant numbers GO-12227, GO-12519, GO-13339, from the Space Telescope Science Institute (operated by AURA, Inc., under NASA contract NAS 5-26555) and Astrophysics Data Analysis Program award "An X-Ray and UV Study of a New Class of AGB stars with Actively-Accreting Binary Companions: fuvAGB Stars" (17-ADAP17-0206)".

Acknowledgments: R.S. would like to thank members of the ESSENcE team (in particular J. Bernard-Salas, J. Cami, M. Matsuura, A. Zijlstra and M. Barlow) for developing the project to observe fullerenes in Tc 1 and UV-irradiated clumps in NGC 6720, that was included as part of an ERO proposal to JWST, and G. Sloan for a discussion on dust in metal-poor environments. R.S.'s contribution to the research described here was carried out at the Jet Propulsion Laboratory (JPL), California Institute of Technology, under a contract with NASA. R.S. is grateful to three anonymous referees whose detailed commentaries have helped improve an earlier version of this paper. (C) 2020 California Institute of Technology. Government sponsorship acknowledged.

Conflicts of Interest: The author declares no conflict of interest.

\section{References}

1. Keaveney, N.; Boyle, L.; Redman, M. Shaping of Planetary Nebulae by Exoplanets. Galaxies 2020, 8, 41. [CrossRef]

2. Boffin, H.M.J.; Jones, D. The Importance of Binaries in the Formation and Evolution of Planetary Nebulae; Springer: Berlin/Heidelberg, Germany, 2019. [CrossRef] 
3. Doan, L.; Ramstedt, S.; Vlemmings, W.H.T.; Mohamed, S.; Höfner, S.; De Beck, E.; Kerschbaum, F.; Lindqvist, M.; Maercker, M.; Paladini, C.; et al. The extended molecular envelope of the asymptotic giant branch star $\pi^{1}$ Gruis as seen by ALMA. II. The spiral-outflow observed at high-angular resolution. Astron. Astrophys. 2020, 633, A13. [CrossRef]

4. Hirano, N.; Shinnaga, H.; Dinh-V-Trung.; Fong, D.; Keto, E.; Patel, N.; Qi, C.; Young, K.; Zhang, Q.; Zhao, J. High-Velocity Bipolar Outflow and Disklike Envelope in the Carbon Star V Hydrae. Astrophys. J. 2004, 616, L43-L46. [CrossRef]

5. Randall, S.K.; Trejo, A.; Humphreys, E.M.L.; Kim, H.; Wittkowski, M.; Boboltz, D.; Ramstedt, S. Discovery of a complex spiral-shell structure around the oxygen-rich AGB star GX Monocerotis. Astron. Astrophys. 2020, 636, A123. [CrossRef]

6. Sánchez Contreras, C.; Alcolea, J.; Bujarrabal, V.; Castro-Carrizo, A.; Velilla Prieto, L.; Santand er-García, M.; Quintana-Lacaci, G.; Cernicharo, J. Through the magnifying glass: ALMA acute viewing of the intricate nebular architecture of $\mathrm{OH}$ 231.8+4.2. Astron. Astrophys. 2018, 618, A164. [CrossRef] [PubMed]

7. Sahai, R.; Trauger, J.T. Multipolar Bubbles and Jets in Low-Excitation Planetary Nebulae: Toward a New Understanding of the Formation and Shaping of Planetary Nebulae. Astron. J. 1998, 116, 1357-1366. [CrossRef]

8. Sahai, R.; Morris, M.; Sánchez Contreras, C.; Claussen, M. Preplanetary Nebulae: A Hubble Space Telescope Imaging Survey and a New Morphological Classification System. Astron. J. 2007, 134, 2200-2225. [CrossRef]

9. Sahai, R.; Morris, M.R.; Villar, G.G. Young Planetary Nebulae: Hubble Space Telescope Imaging and a New Morphological Classification System. Astron. J. 2011, 141, 134. [CrossRef]

10. Sahai, R.; Claussen, M.J.; Schnee, S.; Morris, M.R.; Sánchez Contreras, C. An Expanded Very Large Array and CARMA Study of Dusty Disks and Torii with Large Grains in Dying Stars. Astrophys. J. 2011, 739, L3. [CrossRef]

11. Balick, B.; Frank, A. Shapes and Shaping of Planetary Nebulae. Annu. Rev. Astron. Astrophys. 2002, 40, 439-486. [CrossRef]

12. De Marco, O. The Origin and Shaping of Planetary Nebulae: Putting the Binary Hypothesis to the Test. Publ. Astron. Soc. Pac. 2009, 121, 316. [CrossRef]

13. Blackman, E.G.; Lucchini, S. Using kinematic properties of pre-planetary nebulae to constrain engine paradigms. Mon. Not. R. Astron. Soc. 2014, 440, L16-L20. [CrossRef]

14. García-Segura, G.; López, J.A.; Franco, J. Magnetically Driven Winds from Post-Asymptotic Giant Branch Stars: Solutions for High-Speed Winds and Extreme Collimation. Astrophys. J. 2005, 618, 919-925. [CrossRef]

15. Soker, N. Why Magnetic Fields Cannot Be the Main Agent Shaping Planetary Nebulae. Publ. Astron. Soc. Pac. 2006, 118, 260-269. [CrossRef]

16. García-Segura, G.; Villaver, E.; Langer, N.; Yoon, S.C.; Manchado, A. Single Rotating Stars and the Formation of Bipolar Planetary Nebula. Astrophys. J. 2014, 783, 74. [CrossRef]

17. Sahai, R.; Claussen, M.; Contreras, C.S.; Sanz-Forcada, J.; Guerrero, M.A.; Ortiz, R.; De Marco, O.; Muthumariappan, C. Probing Strong Binary Interactions and Accretion in Asymptotic Giant Branch Stars. Bull. Am. Astron. Soc. 2019, 51, 262.

18. Matthews, L.D.; Claussen, M.J.; Harper, G.M.; Menten, K.M.; Ridgway, S. Unlocking the Secrets of Late-Stage Stellar Evolution and Mass Loss through Radio Wavelength Imaging. Bull. Am. Astron. Soc. 2019, $51,424$.

19. Bond, H.E. Close Binary (and Pulsating) Nuclei of Planetary Nebulae. In IAU Colloq. 53: White Dwarfs and Variable Degenerate Stars; van Horn, H.M., Weidemann, V., Savedoff, M.P., Eds.; Cambridge University Press: Cambridge, UK, 1979; p. 266.

20. Miszalski, B.; Acker, A.; Parker, Q.A.; Moffat, A.F.J. Binary planetary nebulae nuclei towards the Galactic bulge. II. A penchant for bipolarity and low-ionisation structures. Astron. Astrophys. 2009, 505, 249-263. [CrossRef]

21. Miszalski, B.; Acker, A.; Moffat, A.F.J.; Parker, Q.A.; Udalski, A. Binary planetary nebulae nuclei towards the Galactic bulge. I. Sample discovery, period distribution, and binary fraction. Astron. Astrophys. 2009, 496, 813-825. [CrossRef]

22. Jones, D. Planetary Nebulae: What Can They Tell Us about Close Binary Evolution? EAS Publ. Ser. 2015, 71-72, 113-116. [CrossRef]

23. De Marco, O.; Passy, J.C.; Frew, D.J.; Moe, M.; Jacoby, G.H. The binary fraction of planetary nebula central stars-I. A high-precision, I-band excess search. Mon. Not. R. Astron. Soc. 2013, 428, 2118-2140. [CrossRef] 
24. Douchin, D.; De Marco, O.; Frew, D.J.; Jacoby, G.H.; Jasniewicz, G.; Fitzgerald, M.; Passy, J.C.; Harmer, D.; Hillwig, T.; Moe, M. The binary fraction of planetary nebula central stars-II. A larger sample and improved technique for the infrared excess search. Mon. Not. R. Astron. Soc. 2015, 448, 3132-3155. [CrossRef]

25. Miller Bertolami, M.M. New models for the evolution of post-asymptotic giant branch stars and central stars of planetary nebulae. Astron. Astrophys. 2016, 588, A25. [CrossRef]

26. Villaver, E.; Stanghellini, L.; Shaw, R.A. The Mass Distribution of the Central Stars of Planetary Nebulae in the Large Magellanic Cloud. Astrophys. J. 2007, 656, 831-840. [CrossRef]

27. Farihi, J. Circumstellar debris and pollution at white dwarf stars. New Astron. Rev. 2016, 71, 9-34. [CrossRef]

28. Becklin, E.E.; Farihi, J.; Jura, M.; Song, I.; Weinberger, A.J.; Zuckerman, B. A Dusty Disk around GD 362, a White Dwarf with a Uniquely High Photospheric Metal Abundance. Astrophys. J. 2005, 632, L119-L122. [CrossRef]

29. Jura, M. A Tidally Disrupted Asteroid around the White Dwarf G29-38. Astrophys. J. 2003, 584, L91-L94. [CrossRef]

30. Su, K.Y.L.; Chu, Y.H.; Rieke, G.H.; Huggins, P.J.; Gruendl, R.; Napiwotzki, R.; Rauch, T.; Latter, W.B.; Volk, K. A Debris Disk around the Central Star of the Helix Nebula? Astrophys. J. 2007, 657, L41-L45. [CrossRef]

31. Chu, Y.H.; Su, K.Y.L.; Bilikova, J.; Gruendl, R.A.; De Marco, O.; Guerrero, M.A.; Updike, A.C.; Volk, K.; Rauch, T. Spitzer $24 \mu \mathrm{m}$ Survey for Dust Disks around Hot White Dwarfs. Astron. J. 2011, 142, 75. [CrossRef]

32. Bilíková, J.; Chu, Y.H.; Gruendl, R.A.; Su, K.Y.L.; De Marco, O. Spitzer Search for Dust Disks around Central Stars of Planetary Nebulae. Astrophys. J. Suppl. Ser. 2012, 200, 3. [CrossRef]

33. Sahai, R.; Vlemmings, W.H.T.; Nyman, L.A. The Coldest Place in the Universe: Probing the Ultra-cold Outflow and Dusty Disk in the Boomerang Nebula. Astrophys. J. 2017, 841, 110. [CrossRef]

34. Sahai, R.; Nyman, L.A. The Boomerang Nebula: The Coldest Region of the Universe? Astrophys. J. 1997, 487, L155-L159. [CrossRef]

35. Werner, M.W.; Uchida, K.I.; Sellgren, K.; Marengo, M.; Gordon, K.D.; Morris, P.W.; Houck, J.R.; Stansberry, J.A. New Infrared Emission Features and Spectral Variations in NGC 7023. Astrophys. J. Suppl. Ser. 2004, 154, 309-314. [CrossRef]

36. Kamath, D.; Wood, P.R.; Van Winckel, H. Optically visible post-AGB/RGB stars and young stellar objects in the Small Magellanic Cloud: Candidate selection, spectral energy distributions and spectroscopic examination. Mon. Not. R. Astron. Soc. 2014, 439, 2211-2270. [CrossRef]

37. Kamath, D.; Wood, P.R.; Van Winckel, H. Optically visible post-AGB stars, post-RGB stars and young stellar objects in the Large Magellanic Cloud. Mon. Not. R. Astron. Soc. 2015, 454, 1468-1502. [CrossRef]

38. Kamath, D.; Wood, P.R.; Van Winckel, H.; Nie, J.D. A newly discovered stellar type: Dusty post-red giant branch stars in the Magellanic Clouds. Astron. Astrophys. 2016, 586, L5. [CrossRef]

39. De Marco, O.; Izzard, R.G. Dawes Review 6: The Impact of Companions on Stellar Evolution. Publ. Astron. Soc. Aust. 2017, 34, e001. [CrossRef]

40. Iaconi, R.; Reichardt, T.; Staff, J.; De Marco, O.; Passy, J.C.; Price, D.; Wurster, J.; Herwig, F. The effect of a wider initial separation on common envelope binary interaction simulations. Mon. Not. R. Astron. Soc. 2017, 464, 4028-4044. [CrossRef]

41. Shiber, S.; Soker, N. Simulating a binary system that experiences the grazing envelope evolution. Mon. Not. R. Astron. Soc. 2018, 477, 2584-2598. [CrossRef]

42. Arneson, R.A.; Gehrz, R.D.; Woodward, C.E.; Helton, L.A.; Shenoy, D.; Evans, A.; Keller, L.D.; Hinkle, K.H.; Jura, M.; Lebzelter, T.; et al. A SOFIA FORCAST Grism Study of the Mineralogy of Dust in the Winds of Proto-planetary Nebulae: RV Tauri Stars and SRd Variables. Astrophys. J. 2017, 843, 51. [CrossRef]

43. Hillen, M.; de Vries, B.L.; Menu, J.; Van Winckel, H.; Min, M.; Mulders, G.D. The evolved circumbinary disk of AC Herculis: A radiative transfer, interferometric, and mineralogical study. Astron. Astrophys. 2015, 578, A40. [CrossRef]

44. Sahai, R.; Zijlstra, A.; Sánchez Contreras, C.; Morris, M. An Icy, Bipolar Proto-Planetary Nebula with Knotty Jets: IRAS 22036+5306. Astrophys. J. 2003, 586, L81-L85. [CrossRef]

45. Ossenkopf, V.; Henning, T.; Mathis, J.S. Constraints on cosmic silicates. Astron. Astrophys. 1992, 261, 567-578.

46. Artigau, É.; Sivaramakrishnan, A.; Greenbaum, A.Z.; Doyon, R.; Goudfrooij, P.; Fullerton, A.W.; Lafrenière, D.; Volk, K.; Albert, L.; Martel, A.; et al. NIRISS aperture masking interferometry: An overview of science opportunities. In Proceedings of the Space Telescopes and Instrumentation 2014: Optical, Infrared, and Millimeter Wave, Montreal, QC, Canada, 22-27 June 2014; p. 914340. [CrossRef] 
47. Ford, K.E.S.; McKernan, B.; Sivaramakrishnan, A.; Martel, A.R.; Koekemoer, A.; Lafrenière, D.; Parmentier, S. Active Galactic Nucleus and Quasar Science with Aperture Masking Interferometry on the James Webb Space Telescope. Astrophys. J. 2014, 783, 73. [CrossRef]

48. Sahai, R.; Le Mignant, D.; Sánchez Contreras, C.; Campbell, R.D.; Chaffee, F.H. Sculpting a Pre-planetary Nebula with a Precessing Jet: IRAS 16342-3814. Astrophys. J. 2005, 622, L53-L56. [CrossRef]

49. Lagadec, E.; Verhoelst, T.; Mékarnia, D.; Suáeez, O.; Zijlstra, A.A.; Bendjoya, P.; Szczerba, R.; Chesneau, O.; van Winckel, H.; Barlow, M.J.; et al. A mid-infrared imaging catalogue of post-asymptotic giant branch stars. Mon. Not. R. Astron. Soc. 2011, 417, 32-92. [CrossRef]

50. Sahai, R.; Vlemmings, W.H.T.; Gledhill, T.; Sánchez Contreras, C.; Lagadec, E.; Nyman, L.Å.; Quintana-Lacaci, G. ALMA Observations of the Water Fountain Pre-planetary Nebula IRAS 16342-3814: High-velocity Bipolar Jets and an Expanding Torus. Astrophys. J. 2017, 835, L13. [CrossRef]

51. Castro-Carrizo, A.; Bujarrabal, V.; Neri, R.; Alcolea, J.; Sánchez Contreras, C.; Santand er-García, M.; Nyman, L.A. Structure and dynamics of the molecular gas in M 2-9: A follow-up study with ALMA. Astron. Astrophys. 2017, 600, A4. [CrossRef]

52. Ressler, M.E.; Cohen, M.; Wachter, S.; Hoard, D.W.; Mainzer, A.K.; Wright, E.L. The Discovery of Infrared Rings in the Planetary Nebula NGC 1514 During the WISE All-sky Survey. Astron. J. 2010, 140, 1882-1890. [CrossRef]

53. Cami, J.; Bernard-Salas, J.; Peeters, E.; Malek, S.E. Detection of $\mathrm{C}_{60}$ and $\mathrm{C}_{70}$ in a Young Planetary Nebula. Science 2010, 329, 1180. [CrossRef]

54. García-Hernández, D.A.; Manchado, A.; García-Lario, P.; Stanghellini, L.; Villaver, E.; Shaw, R.A.; Szczerba, R.; Perea-Calderón, J.V. Formation of Fullerenes in H-containing Planetary Nebulae. Astrophys. J. 2010, 724, L39-L43. [CrossRef]

55. Guzman-Ramirez, L.; Zijlstra, A.A.; Níchuimín, R.; Gesicki, K.; Lagadec, E.; Millar, T.J.; Woods, P.M. Carbon chemistry in Galactic bulge planetary nebulae. Mon. Not. R. Astron. Soc. 2011, 414, 1667-1678. [CrossRef]

56. Sloan, G.C.; Lagadec, E.; Zijlstra, A.A.; Kraemer, K.E.; Weis, A.P.; Matsuura, M.; Volk, K.; Peeters, E.; Duley, W.W.; Cami, J.; et al. Carbon-rich Dust Past the Asymptotic Giant Branch: Aliphatics, Aromatics, and Fullerenes in the Magellanic Clouds. Astrophys. J. 2014, 791, 28. [CrossRef]

57. Matsuura, M.; Bernard-Salas, J.; Lloyd Evans, T.; Volk, K.M.; Hrivnak, B.J.; Sloan, G.C.; Chu, Y.H.; Gruendl, R.; Kraemer, K.E.; Peeters, E.; et al. Spitzer Space Telescope spectra of post-AGB stars in the Large Magellanic Cloud-polycyclic aromatic hydrocarbons at low metallicities. Mon. Not. R. Astron. Soc. 2014, 439, 1472-1493. [CrossRef]

58. Kwok, S. Organic Synthesis in the Late Stages of Stellar Evolution. EAS Publ. Ser. 2015, 71-72, $349-353$.

59. Gillett, F.C.; Forrest, W.J.; Merrill, K.M. 8-13-micron spectra of NGC 7027, BD +30 3639, and NGC 6572. Astrophys. J. 1973, 183, 87. [CrossRef]

60. Tielens, A.G.G.M. Interstellar polycyclic aromatic hydrocarbon molecules. Annu. Rev. Astron. Astrophys. 2008, 46, 289-337. [CrossRef]

61. Campbell, E.K.; Holz, M.; Gerlich, D.; Maier, J.P. Laboratory confirmation of $\mathrm{C}_{60}{ }^{+}$as the carrier of two diffuse interstellar bands. Nature 2015, 523, 322-323. [CrossRef]

62. Walker, G.A.H.; Campbell, E.K.; Maier, J.P.; Bohlender, D. The 9577 and 9632 A Diffuse Interstellar Bands: $\backslash \mathrm{C}_{60}^{+}$as Carrier. Astrophys. J. 2017, 843, 56. [CrossRef]

63. Linnartz, H.; Cami, J.; Cordiner, M.; Cox, N.L.J.; Ehrenfreund, P.; Foing, B.; Gatchell, M.; Scheier, P. C $60^{+}$as a diffuse interstellar band carrier; a spectroscopic story in 6 acts. J. Mol. Spectrosc. 2020, 367, 111243. [CrossRef]

64. Otsuka, M.; Kemper, F.; Cami, J.; Peeters, E.; Bernard-Salas, J. Physical properties of fullerene-containing Galactic planetary nebulae. Mon. Not. R. Astron. Soc. 2014, 437, 2577-2593. [CrossRef]

65. Hollenbach, D.; Natta, A. Time-dependent Photodissociation Regions. Astrophys. J. 1995, 455, 133. [CrossRef]

66. Cox, N.L.J.; Pilleri, P.; Berné, O.; Cernicharo, J.; Joblin, C. Polycyclic aromatic hydrocarbons and molecular hydrogen in oxygen-rich planetary nebulae: The case of NGC 6720. Mon. Not. R. Astron. Soc. 2016, 456, L89-L93. [CrossRef] [PubMed]

67. Gallino, R.; Arlandini, C.; Busso, M.; Lugaro, M.; Travaglio, C.; Straniero, O.; Chieffi, A.; Limongi, M. Evolution and Nucleosynthesis in Low-Mass Asymptotic Giant Branch Stars. II. Neutron Capture and the S-Process. Astrophys. J. 1998, 497, 388-403. [CrossRef]

(C) 2020 by the author. Licensee MDPI, Basel, Switzerland. This article is an open access article distributed under the terms and conditions of the Creative Commons Attribution (CC BY) license (http:/ / creativecommons.org/licenses/by/4.0/). 